\title{
Radiation Mitigation for Power Line Communications Using Time Reversal
}

\author{
Amilcar Mescco, ${ }^{1}$ Pascal Pagani, ${ }^{2}$ Michel Ney, ${ }^{2}$ and Ahmed Zeddam ${ }^{1}$ \\ ${ }^{1}$ Orange Labs Networks, 2 Avenue Pierre Marzin, 22200 Lannion, France \\ ${ }^{2}$ Telecom Bretagne, Microwave Department, Technopôle Brest-Iroise, 29238 Brest, France
}

Correspondence should be addressed to Amilcar Mescco; amescco@gmail.com

Received 4 August 2012; Revised 16 January 2013; Accepted 21 January 2013

Academic Editor: Moises V. Ribeiro

Copyright (C) 2013 Amilcar Mescco et al. This is an open access article distributed under the Creative Commons Attribution License, which permits unrestricted use, distribution, and reproduction in any medium, provided the original work is properly cited.

Power Line Communication (PLC) is the response for nowadays high demand of multimedia services in domestic environment, not only for its fast and reliable transfer characteristics but also for its flexible low cost implementation, since the PLC technology uses the existing electrical network infrastructure and the omnipresent outlets throughout the home. The transfer of such a high bit rate through the mains network generates acceptable radiated emission regulated by international standards, but the increment in speed for new generation PLC may cause higher levels of emissions. This paper explains the use of the Time Reversal (TR) technique to mitigate radiated emissions from PLC systems. This method was probed experimentally in real electrical networks with excellent results: in $40 \%$ of the observations, the Electromagnetic Interference (EMI) generated by PLC transmission could be reduced by more than $3 \mathrm{~dB}$, and this EMI mitigation factor could increase to more than $10 \mathrm{~dB}$ in particular configurations.

\section{Introduction}

With the increasing demand for both high data rate applications and reliable links for command and control systems, Power Line Communication (PLC) has emerged in recent years as an attractive communication technique [1]. The main advantage of this technology is its ability to benefit from the existing electrical network infrastructure for the transmission of electromagnetic signals. Hence, it becomes possible to build large communication networks without the need for installing new wires.

In the home or office environment, indoor PLC uses the Low Voltage (LV) infrastructure. The presence of several electrical outlets in each room of the house allows ubiquitous coverage of the communication network. In addition, the relatively short distance between different outlets allows the system to operate within a limited attenuation. Current Broad Band (BB) in-home systems primarily operate in the frequency range from $2 \mathrm{MHz}$ to $30 \mathrm{MHz}$. However, recent specifications, such as IEEE 1901 [2] or ITU-T G.9960 [3] allow signal transmission at higher frequencies up to $100 \mathrm{MHz}$. On the other hand, Narrow Band (NB) PLC systems are deployed at frequencies below $500 \mathrm{kHz}$, in both indoor and outdoor configurations, using LV or Medium Voltage (MV) infrastructures [4]. These systems allow the transmission of command and control information over longer distances for Smart Grid applications. The ITU-T G.9955 standard is an example of such NB PLC systems [5].

LV or MV electrical wires were not initially designed to propagate communication signals at frequencies above $1 \mathrm{kHz}$. As a consequence, the communication channel between the transmitter and the receiver is a difficult channel, generating attenuation and multiple propagation paths. The channel capacity is, hence, limited, and signal processing needs to be optimized so as to maximize the offered data rate and Quality of Service (QoS).

This paper focuses on one of the main limitations related to the PLC technology, namely, the generation of unintentional radiated signal. This phenomenon is mainly due to the unbalanced nature of the electrical network [6]. The variation of the impedances of the loads connected to the network as well as the unequal length of the phase and neutral wires (due to single-phase switches) converts the differential PLC signal into common mode current flowing 


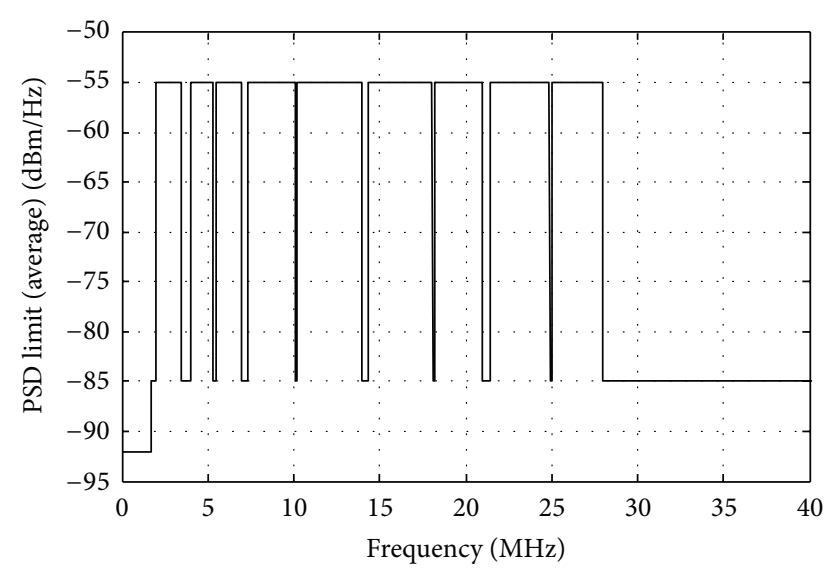

FIgURE 1: Example of PSD limit (average) for North America.

through the network. Consequently, the copper wires used for transmitting the useful signal act as an antenna, and part of the transmitted power is radiated. This not only results in stronger signal attenuation at the receiver but also leads to Electromagnetic Compatibility (EMC) issues, as the radiated signal may interfere with other existing services, such as amateur radio (HAM) or Short Wave (SW) broadcasting. The impact of PLC transmission on EMC has been studied, for example, within the ICT FP7 project OMEGA [7] and through the ETSI Specialist Task Force 410 [8].

In order to avoid interference between PLC systems and other users of the spectrum, regulation authorities impose strict emission masks for the transmission of electromagnetic signals on the power lines. In the USA, the Federal Communications Commission (FCC) Part 15 [9] specifies a maximum level of radiated field for carrier-current systems (including PLC), leading system specifications to define constrained power transmission masks. Figure 1 represents an example of Power Spectral Density (PSD) limits provided in the IEEE 1901 standard for North America. The observed notches are defined to protect specific systems, such as HAM bands. In Europe, CENELEC is currently developing a draft on regulation standard applying to in-home PLC systems [10].

Regardless of the regulation limits in place, the research presented in this paper focuses on the mitigation of unintentional radiation due to PLC systems. Several attempts to solve this problem have been presented in the literature. Reference [11] presents a method to reduce radiated emissions by applying an auxiliary signal cancelling the electromagnetic field on a given point in space. Simulations demonstrated good performance, with the drawback that the Electro Magnetic Interference (EMI) could only be mitigated at a single location. The authors in [12] used additional hardware connected at the wall outlets, in order to reduce asymmetries on power lines, at the cost of an increased complexity of the PLC network.

In our approach, we tried to simultaneously reach two complementary goals by the means of digital signal processing. First, we intend to focus the transmitted signal at the receiver location. The power gain linked to this energy focalization allows in turn relaxing the required power level at the transmitter, hence generating less Electro Magnetic Interference (EMI). Second, we target to reduce the level of energy dissipated at any location except the intended receiver. In particular, it is desirable to minimize the level of radiated power from the electrical wires. These two benefits already appear as features of a known technique in the field of wireless transmission: Time Reversal (TR) [13]. Experimental investigations conducted using Ultra Wide Band (UWB) radio waves demonstrated both the focusing and interference mitigation properties of this technique $[14,15]$.

In this paper, we present for the first time an experimental analysis of TR as a mean to mitigate radiation effects for wired signal transmission. Our investigation focuses on High Frequency (HF) BB PLC signals but could be extended to NB PLC and other wired transmission systems, such as Digital Subscriber Line (DSL) access. The paper is organized as follows. Section 2 presents the concepts of the TR technique and its application to wired systems. Section 3 details the experiment conducted to assess the merits of TR for BB PLC, and the results are statistically analyzed in Section 4. Finally, conclusions are drawn in Section 5.

\section{Time Reversal for Power Line Communications}

2.1. Time Reversal for Wireless Transmission. The TR technique, also known as phase conjugation in the frequency domain, was first used in the fields of acoustics $[16,17]$. More recently, this concept has been successfully extended to electromagnetic waves, where the rich multipath channel provides excellent conditions for its application [13]. The basic concept of TR is simple. Let $\delta(\tau)$ be an ideal, Dirac impulse emitted by a transmitter (Tx) antenna (Figure 2(a)). By definition, at any receiver $(\mathrm{Rx})$ location $\mathbf{r}_{0}$, the received signal is given by the Channel Impulse Response (CIR) $h\left(\tau, \mathbf{r}_{\mathbf{0}}\right)$. The CIR is composed of multiple echoes reflecting the multiple propagation paths of the propagation channel.

TR uses this Channel State Information (CSI) at the Tx to prefilter the signal to be transmitted. More specifically, the CIR $h\left(\tau, \mathbf{r}_{\mathbf{0}}\right)$ is time reversed and normalized to serve as an input filter for the signal to be transmitted (Figure 2(b)). Physically, each delayed echo constituting the TR filter travels, among other multiple paths, through its original propagation path. As a result, the multiple echoes sum up coherently at the receiver, hence focusing the received energy in time.

Mathematically, applying TR leads for any $\mathrm{Rx}$ situated at an arbitrary location $\mathbf{r}$ to the equivalent perceived CIR $h_{\mathrm{TR}}(\tau, \mathbf{r})$ (note that this formulation holds for a real valued CIR) [18]:

$$
h_{\mathrm{TR}}(\tau, \mathbf{r})=\frac{h\left(-\tau, \mathbf{r}_{0}\right)}{\sqrt{\int\left|h\left(\tau, \mathbf{r}_{0}\right)\right|^{2} d \tau}} \otimes h(\tau, \mathbf{r}),
$$

where the symbol $\otimes$ denotes time domain convolution. Formulating (1) in the frequency domain leads to

$$
H_{\mathrm{TR}}(f, \mathbf{r})=\frac{H^{*}\left(f, \mathbf{r}_{0}\right)}{\sqrt{\int\left|H\left(f, \mathbf{r}_{0}\right)\right|^{2} d f}} \times H(f, \mathbf{r}),
$$



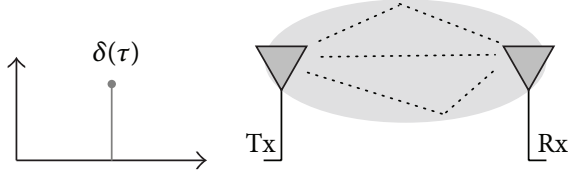

(a)

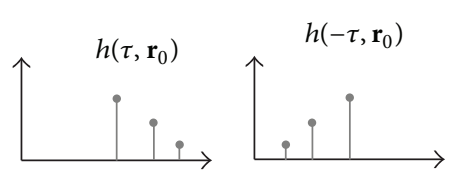

FIGURE 2: Transmission over an ideal multipath propagation channel (a) of a Dirac impulse, (b) of the time-reversed CIR. where $H(f, \mathbf{r})$ represents the complex valued Channel Transfer Function (CTF), and the superscript ${ }^{*}$ denotes the complex conjugate operation. For this reason, TR is sometimes called frequency domain phase conjugation.

Two conclusions can be drawn from (1) and (2). First, at the intended $\mathrm{Rx}$ location $\mathbf{r}_{0}$, the perceived CIR simplifies to

$$
h_{\mathrm{TR}}\left(\tau, \mathbf{r}_{0}\right)=\frac{1}{\sqrt{\int\left|h\left(\tau, \mathbf{r}_{0}\right)\right|^{2} d \tau}} \times R_{h}\left(\tau, \mathbf{r}_{0}\right),
$$

where $R_{h}\left(\tau, \mathbf{r}_{0}\right)$ denotes the time domain autocorrelation of the function $h\left(\tau, \mathbf{r}_{0}\right)$. Similarly, the perceived CTF simplifies to

$$
H_{\mathrm{TR}}\left(f, \mathbf{r}_{0}\right)=\frac{1}{\sqrt{\int\left|H\left(f, \mathbf{r}_{0}\right)\right|^{2} d f}} \times\left|H\left(f, \mathbf{r}_{0}\right)\right|^{2} .
$$

In the time domain, the effect of the TR filter is to transform the CIR into its autocorrelation. For a rich multipath environment, the autocorrelation of the CIR presents a large peak at $\tau=0$, with reduced side echoes. Experimental studies demonstrated that the resulting channel is less spread in time $[14,15]$, hence reducing the possible Intersymbol Interference (ISI). In the frequency domain, the perceived CTF is proportional to the square of the magnitude of the actual CTF. Besides the fact that TR provides a real valued CTF, which could be exploited at the receiver, this also leads to a significant gain in terms of $\mathrm{Rx}$ power, due to a better exploitation of the frequency selective nature of the channel. It was demonstrated in [18] that the application of TR in a flat channel (i.e., without frequency domain power decay) under Rayleigh fading leads to a gain of $3 \mathrm{~dB}$ in the total received power. This gain was increased to $5 \mathrm{~dB}$ when considering the frequency domain power decay observed in practical UWB radio channels.

The second conclusion drawn from (1) and (2) is that, for any other location $\mathbf{r}$ different from $\mathbf{r}_{0}$, TR creates a mismatch between the Tx filter and the channel. This is particularly observable in the frequency domain representation of TR given in (2). The perceived CTF corresponds to the product of two independent CTF, $H(f, \mathbf{r})$ and $H^{*}\left(f, \mathbf{r}_{0}\right)$, with possibly very different frequency fading structures. More precisely, minima of the first CTF can happen randomly at maxima of the second CTF. Hence, averaging over all frequencies, the total received power at untargeted locations is reduced. In wireless TR analysis, this effect is called spatial focusing and is generally assessed as the ratio between the maximum of $h_{\mathrm{TR}}(\tau, \mathbf{r})$ and the maximum of $h_{\mathrm{TR}}\left(\tau, \mathbf{r}_{0}\right)$ for a given distance $\left\|\mathbf{r}-\mathbf{r}_{0}\right\|$. Spatial focusing factors of $-10 \mathrm{~dB}$ have been reported in $[18,19]$.
2.2. Extension of Time Reversal to Wired Transmission. As observed through the study of TR for wireless transmission, the TR scheme provides two main features, namely, an increase of the $\mathrm{Rx}$ power at the intended $\mathrm{Rx}$ location and a decrease of the Rx power at any other location. These features are highly desirable in the context of wired transmission, where the level of Tx power is constrained by the unintentional radiation from the wires, causing possible EMI to other systems.

Based on this observation, we conducted experimental studies to analyze the potential of TR to mitigate unwanted emissions for PLC systems. The main principles of the extension of TR to PLC transmission can be explained with the help of Figure 3.

We assume an intended transmission between a Tx PLC modem and an Rx PLC modem, over an LV indoor electrical network. Different experimental investigations reported the PLC channel as a rich multipath propagation channel, due to the multiple branches present in a classical electrical network and to the impedance mismatch occurring at the network terminations (outlets) and nodes [20-23]. This similarity of the PLC channel with wireless channels suggests promising results when applying TR to PLC.

With reference to Figure 3, the Rx modem is situated at the intended location $\mathbf{r}_{0}$, and the Tx modem is situated at the origin. By applying TR filtering at the Tx, the $\mathrm{Rx}$ power will be increased at location $\mathbf{r}_{0}$; hence, the $\mathrm{Rx}$ modem will benefit from an increased Signal-to-Noise Ratio (SNR). This power increased can also in turn be applied as a reduction of the Tx power to achieve similar performance. At other outlets in the network, situated, for example, at locations $\mathbf{r}_{1}$ or $\mathbf{r}_{2}$, the $\mathrm{Rx}$ power will be reduced. This effect can be further exploited in the design of multiuser transmission schemes.

For our purpose of radiation mitigation, let us now consider a location $\mathbf{r}_{3}$, situated at any point in space in the vicinity of the electrical network. The level of radiated field at this location can be evaluated, for instance, by the means of an equivalent transfer function $H\left(f, \mathbf{r}_{3}\right)$ between the Tx modem and an ideal antenna situated at location $\mathbf{r}_{3}$. By the virtue of the TR scheme (2), the perceived transfer function at location $\mathbf{r}_{3}$ after applying TR will be proportional to the product $H^{*}\left(f, \mathbf{r}_{\mathbf{0}}\right) \times H\left(f, \mathbf{r}_{3}\right)$. As the functions $H^{*}\left(f, \mathbf{r}_{0}\right)$ and $H\left(f, \mathbf{r}_{3}\right)$ are not correlated, their frequency fading structures are different. In particular, the deep notches due to frequency selective fading do not appear at the same frequencies. As a result, the product $H^{*}\left(f, \mathbf{r}_{0}\right) \times H\left(f, \mathbf{r}_{3}\right)$ will provide more average attenuation when compared to $H\left(f, \mathbf{r}_{3}\right)$ alone, and therefore, the total power radiated at location $\mathbf{r}_{3}$ will be reduced. A similar observation is made in previous studies 


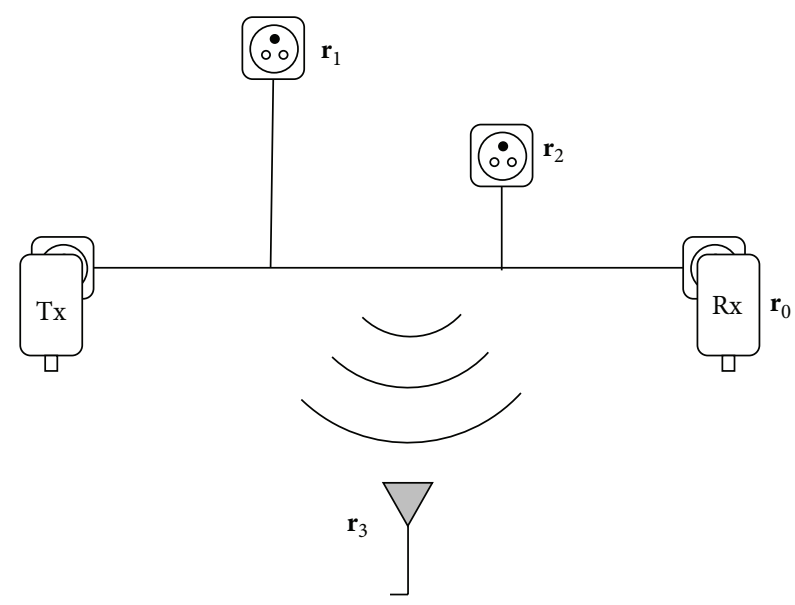

FIgURE 3: Principle of the extension of TR to wired transmission.

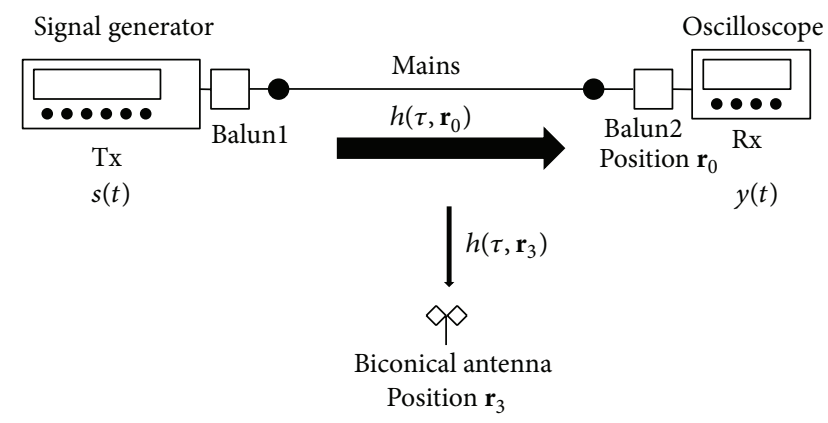

FIGURE 4: Equipment used in the experimental setup.

dedicated to wireless channels $[14,15,18]$. Hence, TR appears as an efficient method to mitigate EMI for wired communications.

The theoretical basis for the application of TR to wired transmission being set, we will now describe the experimental assessment of this method in the next Sections.

\section{Experimental Setup}

3.1. Equipment. In order to experimentally assess the use of TR as a method to mitigate EMI for wired communication, we used the experimental setup presented in Figure 4. In this setup, a signal generator Tektronix AWG7082C was used as a generic Tx. A Digital Sampling Oscilloscope (DSO) LeCroy WaveRunner 715Zi-A was used to sample the received signal at Rx. The signal generated at Tx is denoted $s(t)$, and the signal received at the DSO is denoted $y(t)$. Two Universal PLC Couplers were used as baluns to couple the Tx and Rx signals with the power lines. The Tx coupler is situated at the origin, and the $\mathrm{Rx}$ coupler is connected to a plug at location $\mathbf{r}_{0}$. These couplers were developed within the ETSI Specialist Task Force 410 [24]. In order to measure the power density of the radiated emission received at any arbitrary location $\mathbf{r}_{3}$, a biconical antenna Schwarzbeck EFS921 was connected to a second port of the DSO.

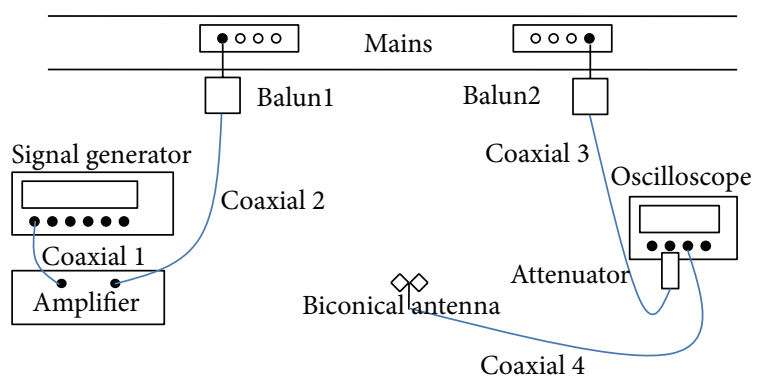

FIgURE 5: Experimental setup calibration.

3.2. Calibration. With reference to Figure 5, two measurement paths can be distinguished. Path 1 serves for the measurement of the CTF and is composed of the following elements: the transmitter, coaxial cable 1 , a $30 \mathrm{~dB}$ amplifier IFIM50, coaxial cable 2, balun 1, mains, balun 2, coaxial cable 3 , a $20 \mathrm{~dB}$ attenuator Radial R412720000, and the receiver. The baluns were considered as part of the channel. The calibration for measurement path 1 was made by directly connecting coaxial cable 2 and coaxial cable 3 .

Path 2 is composed by the transmitter, coaxial cable 1 , a $30 \mathrm{~dB}$ amplifier IFIM50, coaxial cable 2, the wire to free space propagation channel (represented by the CTF $H\left(f, \mathbf{r}_{3}\right)$ ), the biconical antenna, coaxial cable 4, and the DSO. The calibration for this second path was made by connecting coaxial cable 2 and coaxial cable 4 . The gain of the antenna was removed from the measurements by postprocessing.

The signal generator and the DSO were synchronized by a direct connection of their $10 \mathrm{MHz}$ reference clocks. The sampling rates of both devices were set to $f_{s}=100 \mathrm{MHz}$.

3.3. Signal Processing. After initial system calibration, the measurements are performed in 3 steps.

(1) The CTF $H\left(f, \mathbf{r}_{0}\right)$ is evaluated using a specific Tx frame (see below). The considered frequency band extends from $2.8 \mathrm{MHz}$ to $37.5 \mathrm{MHz}$.

(2) The TR filter is generated using the phase and magnitude of $H\left(f, \mathbf{r}_{0}\right)$.

(3) The CTFs $H\left(f, \mathbf{r}_{0}\right)$ and $H\left(f, \mathbf{r}_{3}\right)$ as well as the perceived CTFs $H_{\mathrm{TR}}\left(f, \mathbf{r}_{0}\right)$ and $H_{\mathrm{TR}}\left(f, \mathbf{r}_{3}\right)$ are measured using a single Tx frame (see below). Measurements at the location of the Rx outlet $\left(\mathbf{r}_{0}\right)$ and at arbitrary locations in space $\left(\mathbf{r}_{3}\right)$ are made simultaneously using two ports of the oscilloscope, one connected to the $\mathrm{Rx}$ balun and the other connected to the biconical antenna.

The Tx signal is generated according to the HomePlug standard [25]. The used frame is called PHY Protocol Data Unit (PPDU) and it is composed by a preamble (including a Frame Control) and a number of payload symbols as shown in Figure 6. Each payload symbol consists of a 3072-sample OFDM symbol as defined in the HomePlug specification [25]. In order to estimate the CTFs $H(f, \mathbf{r})$ and $H_{\mathrm{TR}}(f, \mathbf{r})$, the OFDM symbols were loaded with predefined constellations. 


$$
\text { Preamble Symbol } 1 \text { Symbol } 2 \text { Symbol } 3 \text { Symbol } 4 \text { Symbol } 5 \text { Symbol } 6
$$

Figure 6: HomePlug frame.

\begin{tabular}{|c|c|c|c|c|c|c|}
\hline Preamble & $s(t)$ & $s(t)$ & $s(t)$ & $s(t)$ & $s(t)$ & $s(t)$ \\
\hline
\end{tabular}

FIgURE 7: HomePlug frame for calibration and CTF measurement before computation of the TR filter.

The frame used for the calibration and the initial measurement of the CTF before computation of the TR filter is represented in Figure 7.

The frame used for the measurement of the CTF and EMI after computation of the TR filter is represented in Figure 8. Note that in this frame, the TR filter is applied on the 3 last symbols only (represented by the signal $s^{\prime}(t)$ ). From this particular frame scheme, the CTF and EMI can be evaluated with and without application of TR quasi-simultaneously. Recomputing the CTF at this stage also allows monitoring any possible temporal evolution of the channel between calibration and measurement.

In essence, the overall channel estimation process is similar to the channel sounding procedure defined in the HomePlug AV specification. In our experiment, the computation of the CTF from the received signal uses a classical Zero Forcing (ZF) channel estimation procedure. This is suitable in our experiment involving high levels of Signal-toNoise Ratio (SNR) at the Rx. In practical systems operating at lower SNR, more sophisticated methods such as the Minimum Mean Square Error channel estimation would be more efficient against noise enhancement. Note that we chose to actually implement the TR filter in the time domain at the transmitter using a programmable waveform generator, in order to measure results as close as possible to a realistic implementation of the TR scheme in practical PLC modems.

In order to estimate channel attenuation continuously over the measured frequency band, no spectral notches were implemented for this study. In practical systems, a Tx PSD mask is defined, where the Tx power is notched at predefined frequencies, to protect existing services using the same spectrum. The HomePlug specification uses windowing in order to better exploit the power allocated within the PSD mask while protecting out-of-band services. The present study concentrates on reducing the EMI within the band effectively used by PLC systems. Therefore, the results also hold for practical systems including notches.

3.4. Measured Environment. The measurement campaign was conducted using 13 different topologies of $230 \mathrm{~V}$ mains networks within the premises of Orange Labs in Lannion. The campaign took place in different rooms of about $5 \times 4 \mathrm{~m}^{2}$. Figure 9 presents a picture of the experimental setup in an exemplar location.

The Tx and Rx modems were connected to two outlets in the same room, with distances varying between $2 \mathrm{~m}$ and $8 \mathrm{~m}$. In general, the rooms are equipped with several other

\begin{tabular}{|c|c|c|c|c|c|c|}
\hline Preamble & $s(t)$ & $s(t)$ & $s(t)$ & $s^{\prime}(t)$ & $s^{\prime}(t)$ & $s^{\prime}(t)$ \\
\hline
\end{tabular}

FIgURE 8: HomePlug frame for CTF and EMI measurement after application of TR.

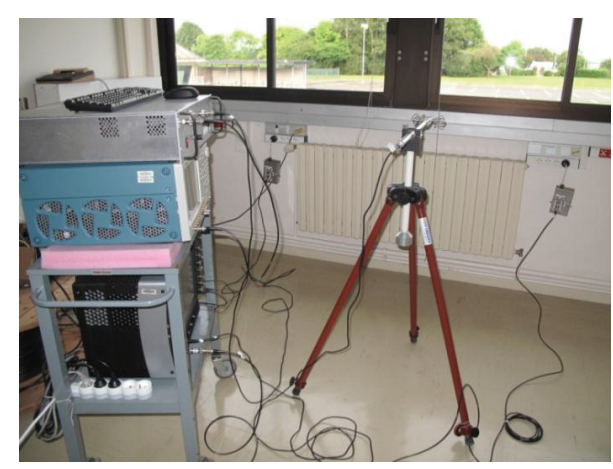

FIGURE 9: Picture of experimentation.

electrical outlets (between 4 and 10). About half of the outlets were connected to classical office appliances (lamps, desktops, etc.). For each topology, one CTF was measured, first without applying TR and then after applying TR filtering. In addition, for each topology, between 3 and 5 locations were selected to measure the received electrical field with the help of the biconical antenna. In total, 13 CTF and 43 measurements of the electrical field were collected for statistical analysis.

\section{Results and Statistical Analysis}

4.1. Preliminary Results. The preliminary results for an exemplar network will be presented in two parts: first, the CTF at $\mathbf{r}_{0}$ and second the electric field and its associated power density at $\mathbf{r}_{3} \neq \mathbf{r}_{0}$.

For the CTF at $\mathbf{r}_{0}$, we show its attenuation characteristics in Figure 10.

Let us first consider the measured CTF (black curve). The deep notches at some frequencies are due to reflections at the terminations of the network and reflect the multipath nature of the PLC network. We define the average attenuation before TR $\overline{H\left(\mathbf{r}_{0}\right)}$ in $\mathrm{dB}$ as:

$$
\overline{H\left(\mathbf{r}_{0}\right)}=10 \log _{10}\left(\frac{1}{f_{\max }-f_{\min }} \int_{f_{\min }}^{f_{\max }}\left|H\left(f, r_{0}\right)\right|^{2} d f\right),
$$

where $f_{\min }$ and $f_{\max }$ respectively represent the minimum and maximum sounded frequencies. The average attenuation corresponds to the signal attenuation perceived by a receiver capable of exploiting all the power received in the frequency band from $f_{\min }$ to $f_{\max }$. Typically, an OFDM system like the HomePlug AV specification is able to exploit the total received power over a wide frequency band. In our example, the average attenuation is about $11 \mathrm{~dB}$.

Let us now focus on the perceived CTF after applying TR. Owing to the mathematical definition of the TR filter, TR allocates more power to frequencies showing minimal 


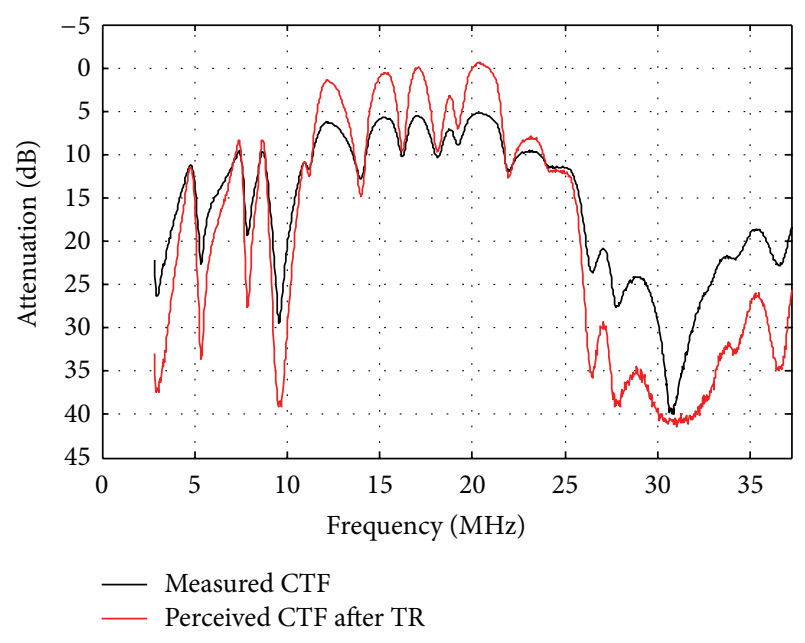

FIGURE 10: Channel attenuation before and after TR.

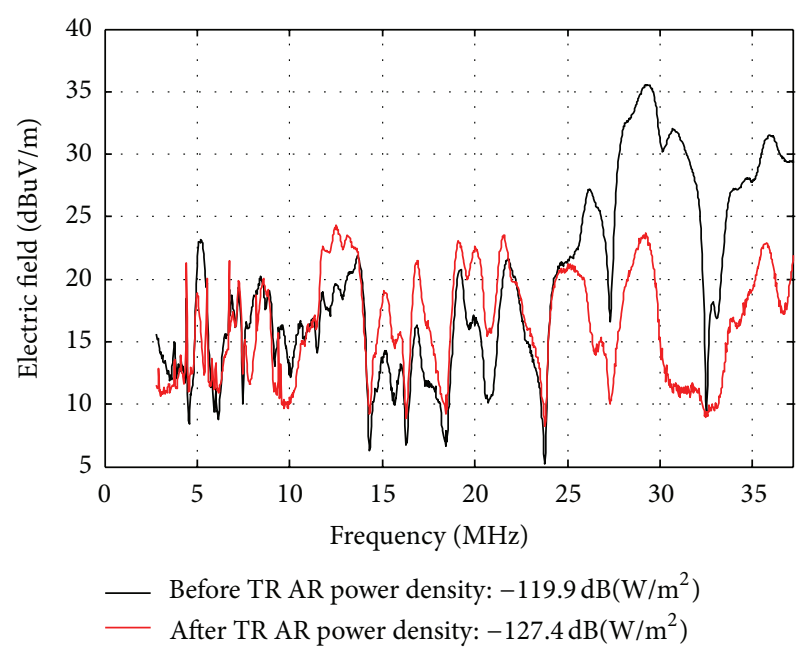

FIGURE 11: Electric field before and after TR.

attenuation, while strongly attenuated frequencies are more power constrained. In particular, for all frequencies where the attenuation of the channel $H\left(f, \mathbf{r}_{0}\right)$ is higher than the average attenuation $\overline{H\left(\mathbf{r}_{0}\right)}$, the perceived channel is more attenuated after applying TR. This can be clearly seen in the frequency range from $26 \mathrm{MHz}$ to $37.5 \mathrm{MHz}$. For the frequencies where the attenuation of the channel is less than $\overline{H\left(\mathbf{r}_{0}\right)}$, the response of the channel is improved using TR. We can see this clearly in the frequency range from $11 \mathrm{MHz}$ to $22 \mathrm{MHz}$. If we define the average attenuation after TR $\overline{H_{\mathrm{TR}}\left(\mathbf{r}_{0}\right)}$ in $\mathrm{dB}$ as

$$
\overline{H_{\mathrm{TR}}\left(\mathbf{r}_{0}\right)}=10 \log _{10}\left(\frac{1}{f_{\max }-f_{\min }} \int_{f_{\min }}^{f_{\max }}\left|H_{\mathrm{TR}}\left(f, r_{0}\right)\right|^{2} d f\right),
$$

we observe that the average attenuation after applying TR is about $7.5 \mathrm{~dB}$. Hence, the application of TR provided a gain in the total received power of $3.5 \mathrm{~dB}$ in this particular example.

Note that only the measured CTF (black curve) is physical. Therefore, the measured CTF will always degrade the transmission with some degree of channel attenuation. On the contrary, the CTF perceived at Rx after application of TR (red curve) corresponds to a logical channel, where the effects of the measured CTF are combined with the effects of the TR filter at Tx. Hence, the perceived CTF may exhibit some gain over a limited frequency range.

We now consider the electrical field and its associated power density at $\mathbf{r}_{3} \neq \mathbf{r}_{0}$. The value of the electrical field $E\left(f, \mathbf{r}_{3}\right)$ in $\mathrm{dB} \mu \mathrm{V} / \mathrm{m}$ was computed from the CTF $H\left(f, \mathbf{r}_{3}\right)$ measured between the Tx balun and the antenna connector assuming an injected PSD $P_{\text {feed }}=-55 \mathrm{dBm} / \mathrm{Hz}$ and using the following formula [24]:

$$
E\left(f, \mathbf{r}_{3}\right)=P_{\text {feed }}+20 \log _{10}\left(\left|H\left(f, \mathbf{r}_{3}\right)\right|\right)+107+\mathrm{AF}(f),
$$

where $\mathrm{AF}(f)$ represents the antenna factor and 107 represents the conversion from $\mathrm{dBm}$ to $\mathrm{dB} \mu \mathrm{V}$. In addition, we computed the average radiated power density $\overline{S\left(\mathbf{r}_{3}\right)}$ in $\mathrm{dB}\left(\mathrm{W} / \mathrm{m}^{2}\right)$ as

$$
\overline{S\left(\mathbf{r}_{3}\right)}=10 \log _{10}\left(\frac{1}{f_{\max }-f_{\min }} \int_{f_{\min }}^{f_{\max }} \frac{1}{120 \pi}\left|E\left(f, \mathbf{r}_{3}\right)\right|^{2} d f\right),
$$

where $f_{\min }$ and $f_{\max }$, respectively, represent the minimum and maximum sounded frequencies, and $E\left(f, \mathbf{r}_{3}\right)$ is expressed in $\mathrm{V} / \mathrm{m}$. In (8), the term $120 \times \pi$ provides the value of the impedance of free space in $\mathrm{Ohm}$.

Note that both $E\left(f, \mathbf{r}_{3}\right)$ and $\overline{S\left(\mathbf{r}_{3}\right)}$ can also be computed after applying TR filtering, using the following equations:

$$
E_{\mathrm{TR}}\left(f, \mathbf{r}_{3}\right)=P_{\text {feed }}+20 \log _{10}\left(\left|H_{\mathrm{TR}}\left(f, \mathbf{r}_{3}\right)\right|\right)+107+\mathrm{AF}(f)
$$

$$
\begin{aligned}
& \overline{S_{\mathrm{TR}}\left(\mathbf{r}_{3}\right)} \\
& \quad=10 \log _{10}\left(\frac{1}{f_{\max }-f_{\min }} \int_{f_{\min }}^{f_{\max }} \frac{1}{120 \pi}\left|E_{\mathrm{TR}}\left(f, \mathbf{r}_{3}\right)\right|^{2} d f\right) .
\end{aligned}
$$

An example of radiated emission measurement is given in Figure 11. For this particular electrical network, the mitigation of radiated emissions is clear in the frequency band from $26 \mathrm{MHz}$ to $37.5 \mathrm{MHz}$, and the average radiated power density has reduced by about $7.5 \mathrm{~dB}$. Thus, we can observe with this example that the application of TR filtering can reduce significantly the level of undesired radiated power.

4.2. Statistical Analysis. In this section, we present the statistical analysis of the measurement database collected within 13 rooms of the office building at Orange Labs in Lannion. The total measurement set is composed of 13 CTF and 43 measurements of the electrical field.

We first computed the channel gain $G_{\mathrm{TR}}$ observed on the perceived CTF $H_{\mathrm{TR}}\left(f, \mathbf{r}_{0}\right)$ after application of TR filtering. As OFDM systems can exploit the total power received over a given frequency band, we computed this gain in $\mathrm{dB}$ for the total received power as

$$
G_{\mathrm{TR}}=\overline{H\left(\mathbf{r}_{0}\right)}-\overline{H_{\mathrm{TR}}\left(\mathbf{r}_{0}\right)} .
$$




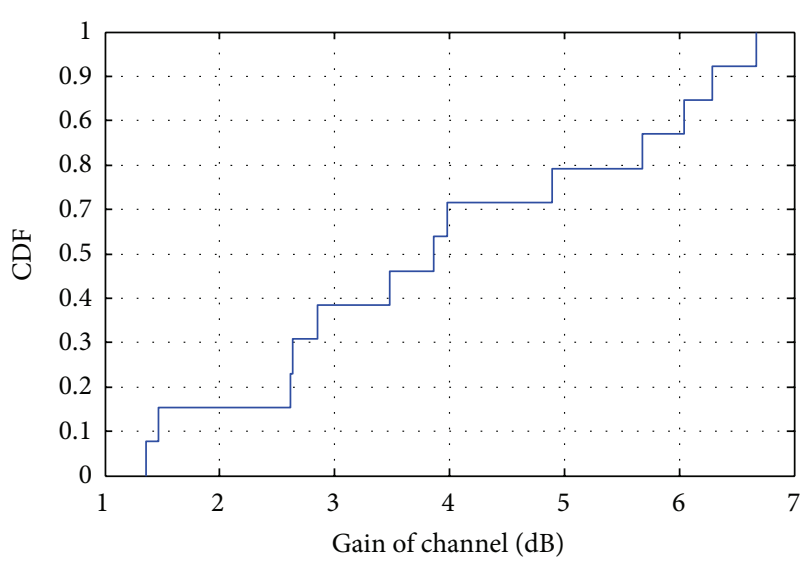

Figure 12: CDF of the channel gain $G_{\mathrm{TR}}$.

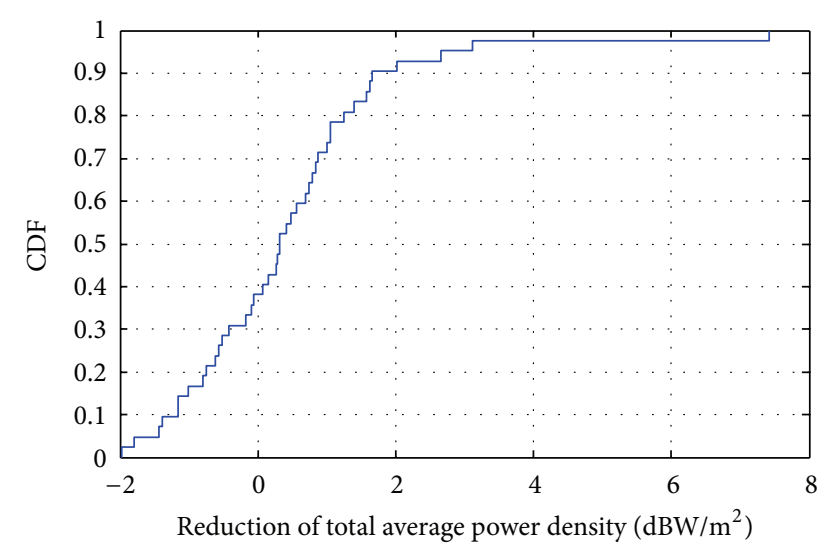

FIgURE 13: CDF of the EMI reduction coefficient $R_{\mathrm{TR}}$.

The cumulative distribution function (CDF) of $G_{\mathrm{TR}}$ is given in Figure 12. This parameter shows always a positive gain, between $1.4 \mathrm{~dB}$ and $6.6 \mathrm{~dB}$ in our experiment, which is in line with results reported in similar wireless experiments [18]. In about $60 \%$ of cases, the channel gain is higher than $3 \mathrm{~dB}$. This means that at $\mathrm{Rx}$ we have always a better reception using TR. This channel gain can in turn be used to reduce the injected PSD at Tx, hence reducing by the same factor the unwanted EMI.

We then computed the EMI reduction coefficient $R_{\mathrm{TR}}$, corresponding to the reduction of the undesired radiated power due to the application of a TR filter. This figure of merit of the reduction of radiated signal is computed in $\mathrm{dB}$ as follows:

$$
R_{\mathrm{TR}}=\overline{S\left(\mathbf{r}_{3}\right)}-\overline{S_{\mathrm{TR}}\left(\mathbf{r}_{3}\right)}
$$

The CDF of the EMI reduction coefficient $R_{\mathrm{TR}}$ is given in Figure 13. Results show that the simple application of TR reduces the EMI in more than $60 \%$ of cases. In the best case, the EMI reduced by more than $7 \mathrm{~dB}$ using TR. In the worst case, the EMI increased by $2 \mathrm{~dB}$. The observations of particular cases indicates that the reduction of EMI is more effective when the CTFs $H\left(f, \mathbf{r}_{0}\right)$ and $H\left(f, \mathbf{r}_{3}\right)$ are highly decorrelated. This is more likely to happen in complex

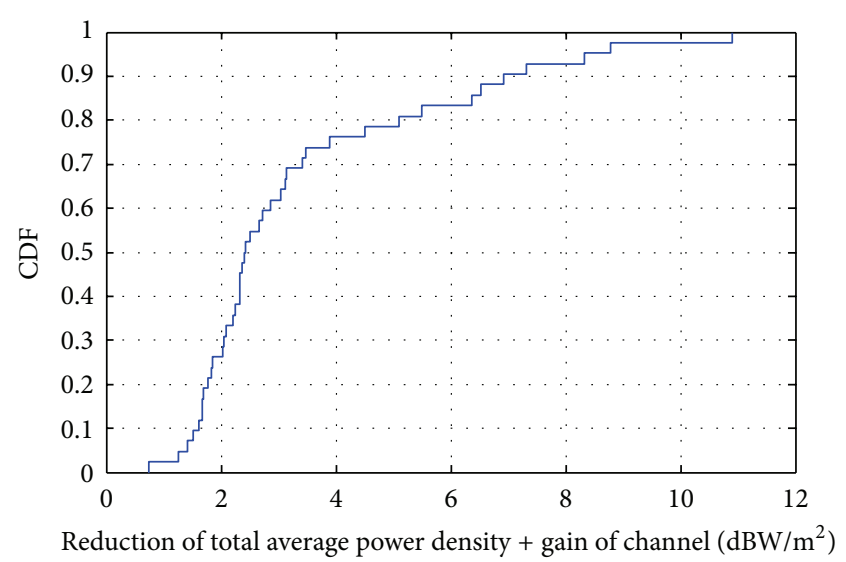

FIGURE 14: CDF of the effective EMI mitigation, $M_{\mathrm{TR}}$.

electrical network topologies, where the rich multipath environment results in different frequency fading structures for different $\mathrm{Rx}$ locations.

Observing the statistics of the channel gain $G_{\mathrm{TR}}$ and of the EMI reduction coefficient $R_{\mathrm{TR}}$, an optimal strategy can be proposed in order to minimize EMI for a PLC system. Indeed, Figure 12 shows that the application of TR provides better system performance due to the reduced channel attenuation. This gives us in turn the flexibility to reduce the level of the Tx power to further reduce EMI while keeping the system performance constant. More precisely, when TR is applied, a Tx power back-off of $G_{\mathrm{TR}} \mathrm{dB}$ can be applied without modifying the total received power. Finally, following this power backoff strategy, the effective EMI mitigation factor $M_{\mathrm{TR}}$ can be computed as the sum of the power backoff and the EMI reduction coefficient:

$$
M_{\mathrm{TR}}=G_{\mathrm{TR}}+R_{\mathrm{TR}}
$$

The CDF of the effective EMI mitigation factor $M_{\mathrm{TR}}$ is depicted in Figure 14. Several conclusions can be drawn from this statistical result.

(i) First, the TR method is able to mitigate EMI generated by PLC transmission in $100 \%$ of our experimental observations. To this respect, one can conclude that the gain $G_{\mathrm{TR}}$ provided by the application of TR allows a Tx power backoff that largely compensates for the possible EMI increment observed in Figure 13.

(ii) Second, in $40 \%$ of the cases, the undesired radiated power is reduced by more than $3 \mathrm{~dB}$.

(iii) Finally, in the most favorable configurations, a reduction of the EMI by more than $10 \mathrm{~dB}$ can be observed. Such configurations correspond to cases where the CTF between the Tx and Rx modem and the EMI spectrum are particularly decorrelated.

\section{Conclusion}

In this paper, we proposed for the first time the application of TR in order to mitigate EMI generated by wired communication systems. TR was originally used in the field of wireless 
transmission as a mean to focus the transmitted signal in both time and space around the intended receiver. We proposed to use the same property to focus the signal injected in a wired medium, such as the electrical network in the case of PLC for instance. As a result, the energy lost through undesired radiation is expected to decrease significantly.

We presented an experimental setup in order to demonstrate this method. The experiment was conducted in the time domain using signal frames similar to the industrial specification HomePlug AV. In addition, the TR filter was actually implemented using an arbitrary wave generator, thus providing results encompassing the possible drawbacks of a practical implementation.

Results demonstrated that, on the wired medium, TR could provide a transmission channel gain between $1 \mathrm{~dB}$ and $7 \mathrm{~dB}$, which is similar to the gain observed in wireless transmission. In addition, the application of a TR filter alone could effectively reduce EMI for $60 \%$ of the observations, with a maximum EMI mitigation of $7 \mathrm{~dB}$. Finally, by combining the channel gain and the EMI reduction features, we experimentally demonstrated that TR was efficient in $100 \%$ of the observed cases to reduce EMI. In $40 \%$ of the cases, the EMI mitigation was larger than $3 \mathrm{~dB}$, with maxima higher than $10 \mathrm{~dB}$. TR is, thus, seen as a promising technique to help resolving EMC issues related to PLC and other wired media.

In the TR strategy presented in this paper, the CTF gain provided by the TR technique was fully dedicated as a power backoff to minimize the EMI. Another strategy could target an increase of the offered capacity while maintaining a constant EMI. Further analyses will, therefore, be dedicated to analyze the tradeoff between channel throughput increase and EMI reduction. In addition, future research will focus on the study of wired TR at higher frequencies and on other media, such as DSL cables. Finally, optimal protocols will be developed to practically implement TR in future standards. In particular, the application of TR to multicast or broadcast scenarios, involving one Tx modem and several $\mathrm{Rx}$ modems, could be further investigated.

\section{References}

[1] H. C. Ferreira, L. Lampe, J. Newbury, and T. G. Swart, Eds., Power Line Communications: Theory and Applications for Narrowband and Broadband Communications Over Power Lines, Wiley, Chichester, UK, 2010.

[2] IEEE, 1901-2010, "IEEE standard for broadband over power line networks: medium access control and physical layer specifications," 2010.

[3] ITU-T G. 9960, "Unified high-speed wireline-based home networking transceivers-system architecture and physical layer specification," 2010.

[4] S. Galli, A. Scaglione, and Z. Wang, "For the grid and through the grid: the role of power line communications in the smart grid," Proceedings of the IEEE, vol. 99, no. 6, pp. 998-1027, 2011.

[5] V. Oksman and J. Zhang, "G.HNEM: the new ITU-T standard on narrowband PLC Technology," IEEE Communications Magazine, vol. 49, no. 12, pp. 36-44, 2011.

[6] M. Ishihara, D. Umehara, and Y. Morihiro, "The correlation between radiated emissions and power line network components on indoor power line communications," in Proceedings of the IEEE International Symposium on Power Line Communications and Its Applications (ISPLC '06), pp. 314-318, Orlando, Fla, USA, March 2006.

[7] Seventh Framework Programme: Theme 3 ICT-213311 OMEGA, Deliverable D3.3, "Report on Electro Magnetic Compatibility of Power Line Communications", 2009.

[8] A. Schwager, W. Bäschlin et al., "European MIMO PLC Field Measurements: overview of the ETSI STF410 Campaign \& EMI Analysis," in IEEE International Symposium on Power Line Communications and Its Applications (ISPLC '12), pp. 304-309, Beijing, China, March 2012.

[9] Federal Communications Commission, "Title 47 of the code of federal regulations part 15," 2007.

[10] CENELEC, Final Draft European Standard FprEN 50561-1, "Power line communication apparatus used in low voltage installations-Radio disturbance characteristics-Limits and methods of measurement-Part 1: Apparatus for in-home use" 2011.

[11] A. Vukicevic, M. Rubinstein, F. Rachidi, and J. L. Bermudez, "On the impact of mitigating radiated emissions on the capacity of PLC systems," in Proceedings of the IEEE International Symposium on Power Line Communications and Its Applications (ISPLC '07), pp. 487-492, Pisa, Italy, March 2007.

[12] P. Favre, C. Candolfi, and P. Krahenbuehl, "Radiation and disturbance mitigation in PLC networks," in Proceedings of the 20th International Zurich Symposium on Electromagnetic Compatibility (EMC Zurich '09), pp. 5-8, Zürich, Switzerland, January 2009.

[13] G. Lerosey, J. De Rosny, A. Tourin, A. Derode, G. Montaldo, and M. Fink, "Time reversal of electromagnetic waves," Physical Review Letters, vol. 92, no. 19, Article ID 193904, 4 pages, 2004.

[14] A. E. Akogun, R. C. Qiu, and N. Guo, "Demonstrating the leverages of time reversal in ultra-wideband communications using time domain measurements," in Proceedings of the 51st International Instrumentation Symposium, pp. 737-742, Knoxville, Tenn, USA, May 2005.

[15] A. Khaleghi, G. El Zein, and I. H. Naqvi, "Demonstration of time-reversal in indoor ultra-wideband communication: time domain measurement," in Proceedings of the 4th IEEE International Symposium on Wireless Communication Systems (ISWCS '07), pp. 465-468, October 2007.

[16] A. Derode, P. Roux, and M. Fink, "Acoustic time-reversal through high-order multiple scattering," in Proceedings of the IEEE International Ultrasonics Symposium, vol. 2, pp. 1091-1094, Seattle, Wash, USA, November 1995.

[17] D. R. Jackson and D. R. Dowling, "Phase conjugation in underwater acoustics," Journal of the Acoustical Society of America, vol. 89, pp. 171-181, 1991.

[18] P. Pajusco and P. Pagani, "On the use of uniform circular arrays for characterizing UWB time reversal," IEEE Transactions on Antennas and Propagation, vol. 57, no. 1, pp. 102-109, 2009.

[19] C. Zhou and R. C. Qiu, "Spatial focusing of time-reversed UWB electromagnetic waves in a hallway environment," in Proceedings of the 38th Southeastern Symposium on System Theory, pp. 318-322, Cookeville, Tenn, USA, March 2006.

[20] M. Tlich, A. Zeddam, F. Moulin, and F. Gauthier, "Indoor power-line communications channel characterization up to $100 \mathrm{MHz}$ - part I: one-parameter deterministic model," IEEE Transactions on Power Delivery, vol. 23, no. 3, pp. 1392-1401, 2008.

[21] M. Tlich, A. Zeddam, F. Moulin, and F. Gauthier, "Indoor power-line communications channel characterization up to 100 
MHz-part II: time-frequency analysis," IEEE Transactions on Power Delivery, vol. 23, no. 3, pp. 1402-1409, 2008.

[22] A. M. Tonello and F. Versolatto, "Bottom-up statistical PLC channel modeling-part I: random topology model and efficient transfer function computation," IEEE Transactions on Power Delivery, vol. 26, no. 2, pp. 891-898, 2011.

[23] A. M. Tonello and F. Versolatto, "Bottom-up statistical PLC channel modeling - part II: inferring the statistics," IEEE Transactions on Power Delivery, vol. 25, no. 4, pp. 2356-2363, 2010.

[24] ETSI TR 101 562-1 V2.1.1, "Powerline telecommunications (PLT), MIMO PLT, part 1: measurements methods of MIMO PLT," Tech. Rep., 2012, Chapter 7.1.

[25] HomePlug, "HomePlug AV specification, version 1.1," 2007. 

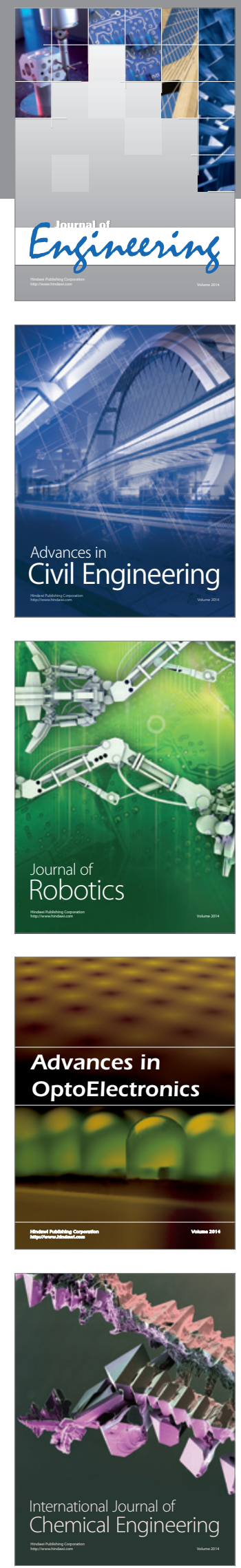

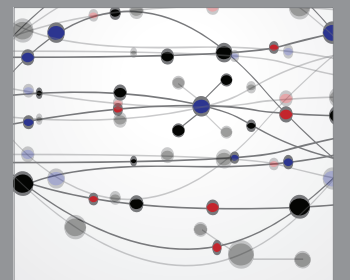

The Scientific World Journal
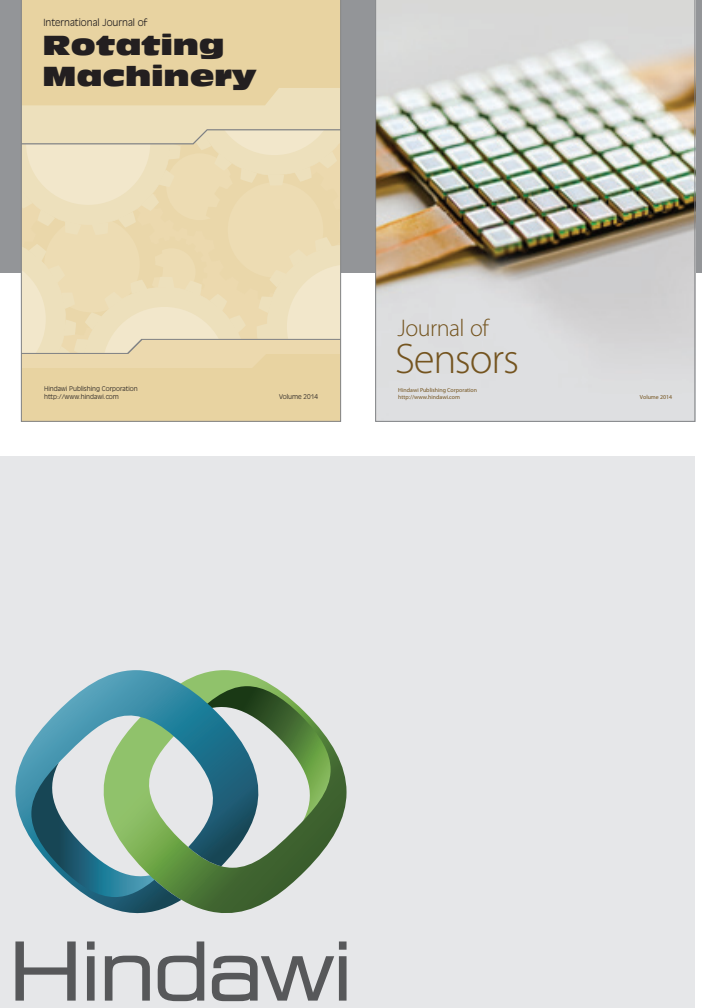

Submit your manuscripts at http://www.hindawi.com
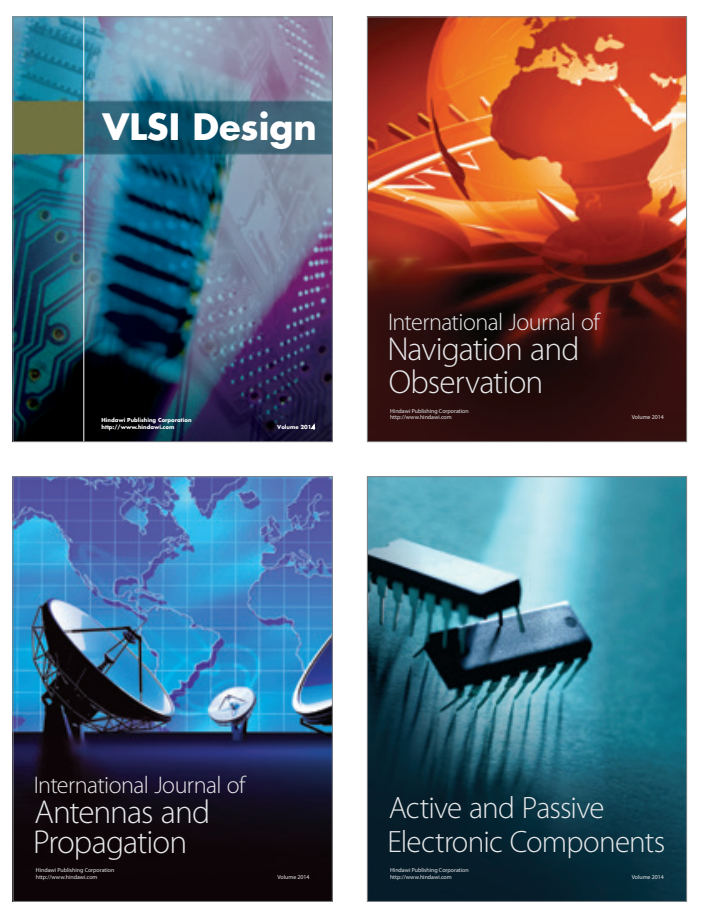
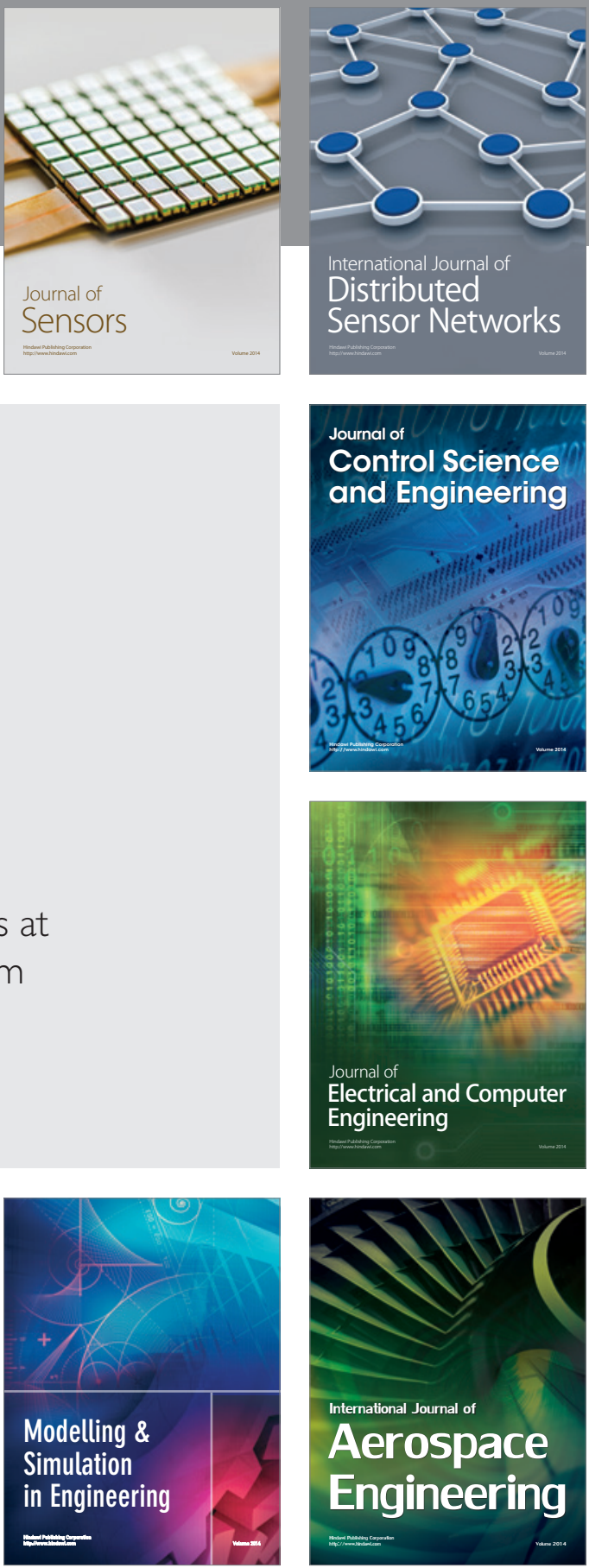

Journal of

Control Science

and Engineering
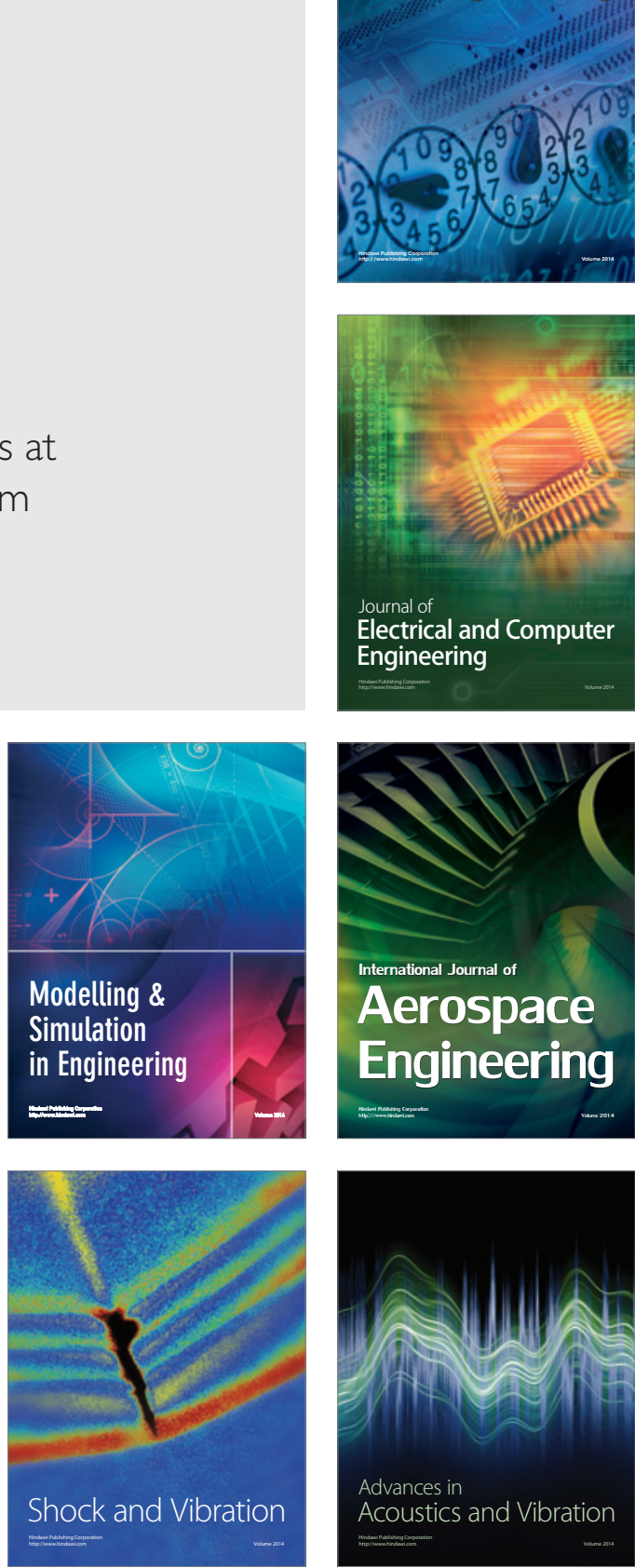